\title{
Detecting diabetic risk using the oral glucose tolerance test in Chinese patients with hypertension: a cross-sectional study
}

\author{
Ningling Sun ${ }^{1}$, Hongyi Wang ${ }^{1}$, Yuemin Sun ${ }^{2}$, Xiaoping Chen ${ }^{3}$, Hao Wang ${ }^{4}$, Lianyou Zhao ${ }^{5}$, Jinping Wang ${ }^{6}$ \\ and Zhiming $\mathrm{Zhu}^{7}$
}

To analyze the clinical significance of the oral glucose tolerance test (OGTT) in the assessment of abnormal glucose metabolism in Chinese patients with hypertension. In this cross-sectional study of 10173 hypertensive adult patients from eight sites in China, data on patient, clinical and disease characteristics were collected, and fasting plasma glucose (FPG) levels were measured. A subset of 5322 patients also underwent an OGTT. Abnormal glucose metabolism (impaired glucose regulation (IGR) or diabetes mellitus (DM)) was detected in $31.1 \%$ of the 10173 patients by FPG testing and in $67 \%$ of the 5322 patients by OGTT. OGTT increased the detection rate by $35.8 \%$. Patients with FPG levels $\geqslant 5.6$ and $\geqslant 6.1 \mathrm{mmol}^{-1}$ had the mean OGTT 2-h plasma glucose levels of 8.79 and $9.68 \mathrm{mmolI}^{-1}$, respectively. Compared with normoglycemic patients, those with IGR or DM had higher rates of total proteinuria (normoglycemic $15.8 \%$ vs. IGR $22.1 \%$ vs. DM $33.7 \%$, $P<0.001)$, a lower absolute estimated glomerular filtration rate (eGFR; in $\mathrm{ml} \mathrm{min}^{-1}$ per $\left.1.73 \mathrm{~m}^{2}\right)(82.9$ vs. 80.77 vs. 79.74 , $P=0.06$ ) and a higher prevalence of cardiovascular disease (normoglycemic $17.6 \%$ vs. IGR/DM $13.8 \%, P<0.001$ ). Abnormal abdominal circumference, eGFR $\leqslant 60 \mathrm{ml} \mathrm{min}^{-1}$ per $1.73 \mathrm{~m}^{2}$, and proteinuria were independently associated with abnormal glucose metabolism (IGR or DM). Hypertensive patients who are diabetic or at risk of diabetes are at greater risk of renal damage and cardiovascular disease than those who are normoglycemic. It is insufficient to assess the glucose metabolism status of Chinese hypertensive patients using only FPG testing; the use of OGTT can increase the detection rate by $35.8 \%$. Patients whose FPG levels were $<5.6 \mathrm{mmol} \mathrm{I}^{-1}$ may be found to have abnormal glucose metabolism after an OGTT.

Hypertension Research (2014) 37, 82-87; doi:10.1038/hr.2013.126; published online 17 October 2013

Keywords: diabetes mellitus; fasting plasma glucose; hypertension; impaired glucose regulation; oral glucose tolerance test

\section{INTRODUCTION}

Currently, the number of hypertensive patients in China is $200000000 .{ }^{1}$ The number of premature deaths from cardiovascular and cerebrovascular diseases due to hypertension has reached $\sim 2330000 ; ;^{2}$ therefore, hypertension is now the leading cause of death in China and, therefore, a risk to the health of the Chinese population.

Diabetes mellitus (DM) is one of the most important risk factors for hypertension, and people with both DM and hypertension have approximately twice the risk of cardiovascular disease as non-diabetic people with hypertension. ${ }^{3}$ Impaired glucose regulation (IGR; 'pre-diabetes') is a critical intermediate stage in the development of DM. ${ }^{4}$ The China Heart Survey showed that the prevalence of abnormal glucose metabolism in patients with coronary artery disease would be underestimated using fasting plasma glucose (FPG) testing alone. Specifically, $87.4 \%$ of patients with IGR and $80.5 \%$ of patients with DM would have remained undiagnosed if oral glucose tolerance tests (OGTTs) had not been performed. ${ }^{5}$ It is not clear whether the risk of DM development in hypertensive patients in China can be comprehensively assessed only with FPG testing. Owing to the importance of assessing the true glucose metabolism status of hypertensive patients, we designed this cross-sectional study to compare the FPG and OGTT tests in screening for pre-diabetes and DM in patients with hypertension. These results are expected to be meaningful because of the large numbers of Chinese patients with hypertension who may also have diabetes.

${ }^{1}$ Heart Center, Peking University People's Hospital, Beijing, China; ${ }^{2}$ Department of Cardiology, Tianjin Medical University General Hospital, Tianjin, China; ${ }^{3}$ Department of Cardiology, West China Hospital of Sichuan University, Chengdu, China; ${ }^{4}$ Department of Hypertension, Henan Provincial People's Hospital, Zhengzhou, China; ${ }^{5}$ Department of Cardiology, Tangdu Hospital of The Fourth Military Medical University, Chongqing, China; ${ }^{6}$ Department of Cardiology, Daqing Oilfield General Hospital of Heilongjiang Province, Daqing, China and ${ }^{7}$ Department of Hypertension and endocrinology, Daping Hospital of Chongqing, Chongqing, China

Correspondence: N Sun, Heart Center, Peking University People's Hospital, No. 11, Xi-Zhi-Men South Street, Beijing 100044, China.

E-mail: nlsun@263.net

Received 21 January 2013; revised 29 May 2013; accepted 28 June 2013; published online 17 October 2013 


\section{METHODS}

\section{Subjects}

Patients diagnosed with essential hypertension (regardless of whether they were receiving antihypertensive therapy) and those with 18-75 years of age were enrolled. All patients included in the study agreed to undergo fasting blood tests; a subset of patients also agreed to undergo an OGTT. The diagnosis of hypertension complied with the 2010 Chinese hypertension guidelines. ${ }^{1}$ The study excluded patients with secondary hypertension but did not exclude patients with a history of cardiovascular or cerebrovascular disease.

\section{Study design}

This was a cross-sectional study. Large hospitals ( $>2000$ inpatient beds) from the east, west, south, north and central areas of China were selected to participate; community hospitals affiliated with each of these hospitals also participated. One primary investigator and two subinvestigators were based at each hospital, all of whom were trained according to a uniform protocol. Investigators at each hospital enrolled outpatients from cardiology, hypertension and general internal medicine wards of the large hospitals and general outpatients from the associated community hospitals between 1 August 2007 and 31 December 2008.

All patients who met the inclusion criteria and who read and signed an informed consent statement were included in the study. On the day of enrollment, study investigators completed the survey questionnaire based on verbal responses from patients, and they conducted a physical examination of the patient. The questionnaire captured the patient's history of hypertension, use of antihypertensive agents, risk factors for cardiovascular diseases and presence of cardiovascular and cerebrovascular diseases. The physical examination included measuring blood pressure (BP), body mass index and abdominal circumference. Blood and urine samples were collected from patients on the day after their questionnaire/physical examinations to screen for DM and IGR using FPG and for albuminuria and proteinuria (see Study assessments). In addition, those patients who had signed an informed consent statement to undergo an OGTT were then tested using this method.

A seated BP was measured in the clinic by trained technicians using a mercury sphygmomanometer after patients had rested quietly for $5 \mathrm{~min}$. The systolic BP (SBP) and diastolic BP (DBP) were recorded at the first appearance (phase I) and the disappearance (phase V) of Korotkoff sounds. An average value was obtained from three separate measurements with an interval of $2 \mathrm{~min}$.

All tests of blood biochemistry (including FPG and lipids) were conducted in certified laboratories that meet the national standards (each participating hospital in this study has a laboratory that meets the national standards). Blood was collected after $12 \mathrm{~h}$ of fasting. A simplified OGTT was conducted, in which venous plasma glucose levels were measured $2 \mathrm{~h}$ after oral intake of $350 \mathrm{ml}$ water with $75 \mathrm{~g}$ anhydrous glucose powder; this plasma glucose level was referred to as the 2-h plasma glucose level.

Venous blood was collected and the serum creatinine ( $\mathrm{SCr}$ ) was measured using the picric acid method at each study site. The estimated glomerular filtration rate (eGFR) was determined using the following modified MDRD formula: ${ }^{6} \mathrm{eGFR}=186 \times \mathrm{SCr}^{-1.154} \times \mathrm{Age}^{0.203} \times 0.742($ female $) \times 1.233$. In the formula, eGFR units are $\mathrm{ml} \mathrm{min}^{-1}$ per $1.73 \mathrm{~m}^{2}$; SCr units are $\mathrm{mg} \mathrm{dl}^{-1}$; weight is in $\mathrm{kg}$; and age is in years.

Standard urine tests for proteinuria were conducted in all patients. Urinary albumin/creatinine ratios were measured with microalbuminuria test paper provided by the Bayer HealthCare Company in patients in whom proteinuria was not detected by this method. Microalbuminuria was defined as an abnormal urinary albumin/creatinine ratio, and clinical proteinuria was defined as urinary protein levels $>0.3 \mathrm{gl}^{-1}$.

An FPG level $\geqslant 7.0 \mathrm{mmoll}^{-1}$ or a 2 -h plasma glucose level $\geqslant 11.1 \mathrm{mmoll}^{-1}$ was the diagnostic of DM. IGR was diagnosed when the FPG was $\geqslant 5.6$ and $<7.0 \mathrm{mmoll}^{-1}$ (impaired fasting glucose, IFG), when the 2-h plasma glucose level was $\geqslant 7.8$ and $<11.1 \mathrm{mmoll}^{-1}$ (impaired glucose tolerance, IGT) or when patients had both an abnormal IFG and IGT (in the subset of patients who had both an FPG test and an OGTT). ${ }^{7}$
According to the Chinese guidelines for hypertension management, ${ }^{1}$ the definitions of the normal ranges of some parameters in our study were as follows: BP: SBP $<140 \mathrm{mmHg}$, DBP $<90 \mathrm{~mm} \mathrm{Hg}$; body mass index $<24 \mathrm{~kg} \mathrm{~cm}^{-2}$; abdominal circumference $<90 \mathrm{~cm}$ (men) and $<85 \mathrm{~cm}$ (women); and a low-density lipoprotein cholesterol $<3.3 \mathrm{mmoll}^{-1}$. The normal range for FPG was defined according to the American Diabetes Association ( $\left.\mathrm{FPG}<5.6 \mathrm{mmoll}^{-1}\right)$.

This study was approved by the Ethics Committee of Peking University People's Hospital, as well as by each of the participating hospitals (see Acknowledgments for the full list of all participating hospitals).

\section{Study objectives}

The study objectives included identifying the following: (1) the proportion of patients with IFG or DM according to the FPG criteria; (2) the proportion of patients with IGT or DM according to the OGTT criteria; (3) the relationship between BP control and glucose regulation; and (4) the relationship between glucose metabolism and proteinuria.

\section{Statistical methods}

The Epidata software (Epidata 3.0 for Microsoft Windows) was used to double enter and validate the data. Data analysis was performed using SPSS 11.5 for Windows. The mean \pm s.d. was calculated for continuous variables. Between-group comparisons were made using analysis of variance (ANOVA) for continuous data and the Wilcoxon rank sum test for categorical data. Factors associated with abnormal glucose metabolism were analyzed through logistic regression analysis. A $P$-value of $<0.05$ was defined as statistically significant. Missing data were substituted with the mean value of each item.

\section{RESULTS}

A total of eight study sites and 37 hospitals were involved in the study; of these, 26 were large hospitals and 11 were community hospitals.

\section{Subjects}

A total of 10212 patients were eligible for inclusion and completed the questionnaires, blood tests and urine tests. Of these, 39 patients did not have FPG samples and were excluded. Therefore, a total of 10173 patients were enrolled in the study. There were 5097 men and 5076 women, and 6382 patients were in large hospitals and 3791 in community hospitals. Their mean age, SBP, DBP, body mass index, FPG, total cholesterol, triglyceride, LDL-C, high-density lipoprotein cholesterol and SCr levels were $61.71 \pm 11.57$ years, $141.47 \pm$ $17.64 \mathrm{~mm} \mathrm{Hg}, \quad 85.08 \pm 11.95 \mathrm{~mm} \mathrm{Hg}, \quad 25.21 \pm 3.43 \mathrm{~kg} \mathrm{~m}^{-2}, \quad 5.41 \pm$ $1.57 \mathrm{mmoll}^{-1}, \quad 4.90 \pm 1.06 \mathrm{mmoll}^{-1}, \quad 1.83 \pm 1.31 \mathrm{mmoll}^{-1}, \quad 3.17$ $\pm 0.95 \mathrm{mmoll}^{-1}, 1.22 \pm 0.37 \mathrm{mmoll}^{-1}$ and $84.46 \pm 28.82 \mu \mathrm{moll}^{-1}$, respectively.

\section{FPG test results}

FPG levels were $<5.6 \mathrm{mmoll}^{-1}$ in 7006 hypertensive patients (68.9\% of the 10173 patients), $>5.6 \mathrm{mmoll}^{-1}$ in 3167 patients $(31.2 \%), \quad 5.6-7.0 \mathrm{mmoll}^{-1}$ in 2611 patients $(25.7 \%)$ and $>7.0 \mathrm{mmoll}^{-1}$ in 556 patients (5.5\%). Figure 1 shows that $31.2 \%$ of the hypertensive patients were diagnosed with IFG or DM using the FPG.

The BP control rates of patients with IGR or DM (as determined using FPG tests) were clearly lower than those of normoglycemic patients (SBP control rate of $41.0 \%$ vs. $46.4 \%$; DBP control rate of $57.7 \%$ vs. 62.1\%; Mann-Whitney test, both $P<0.001$ ). See Table 1 for the proportion of patients in each group who met the guideline criteria for other risk factors of cardiovascular diseases. 


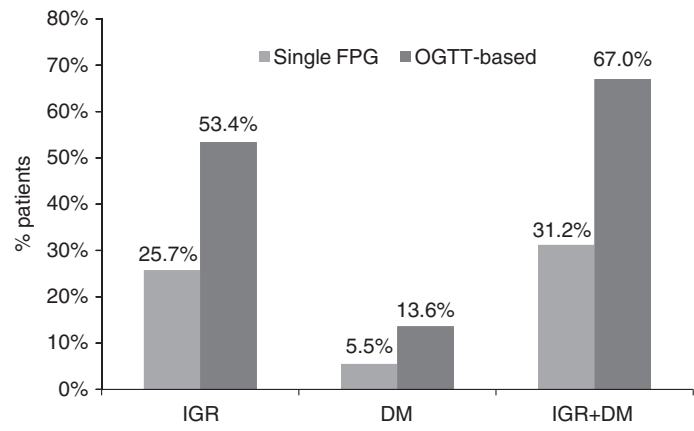

Figure 1 Proportion of hypertensive patients with abnormal glucose metabolism, as detected using either the FPG criteria (blue) or the 2-h OGTT criteria (red). sFPG, single fasting plasma glucose testing; OGTTbased, OGTT-based blood glucose testing; IGR, impaired glucose regulation; DM, diabetes mellitus. A full color version of this figure is available at the Hypertension Research journal online.

Table 1 Diabetes-related clinical and biochemical variables and control rate (proportion of subjects with parameter within the normal range $^{a}$ ) according to the guidelines ${ }^{1,7}$ for these variables, stratified by glucose metabolism status determined by fasting plasma glucose (FPG) in 10173 patients with hypertension

\begin{tabular}{lcccc}
\hline & $\begin{array}{c}\text { Normal glucose } \\
\text { metabolism } \\
\mathrm{n}=7006\end{array}$ & $\begin{array}{c}\text { Control } \\
\text { rate } \\
\text { (\% pts) }\end{array}$ & $\begin{array}{c}\text { Abnormal glucose } \\
\text { metabolism } \\
\mathrm{n}=3167\end{array}$ & $\begin{array}{c}\text { Control } \\
\text { rate } \\
\text { (\% pts) }\end{array}$ \\
\hline SBP (mm Hg) & $140.83 \pm 17.51$ & 46.4 & $142.89 \pm 17.84^{\mathrm{b}}$ & $41.0^{\mathrm{c}}$ \\
DBP (mm Hg) & $84.75 \pm 11.86$ & 62.1 & $85.80 \pm 12.12^{\mathrm{b}}$ & $57.7^{\mathrm{c}}$ \\
BMI (kg m $\left.{ }^{-2}\right)$ & $24.99 \pm 3.42$ & 38.1 & $25.69 \pm 3.40^{\mathrm{b}}$ & $30.6^{\mathrm{c}}$ \\
Abdominal & $86.98 \pm 9.94$ & 49.9 & $89.43 \pm 10.23^{\mathrm{b}}$ & $40.4^{\mathrm{c}}$ \\
circumference & & & & \\
(cm) & & & & \\
LDL-C (mmol I-1) & $3.13 \pm 0.94$ & 60.2 & $3.25 \pm 0.98^{\mathrm{b}}$ & $54.4^{\mathrm{c}}$ \\
FPG (mmol I-1) & $4.90 \pm 0.45$ & NA & $6.54 \pm 2.37^{\mathrm{b}}$ & $\mathrm{NA}$
\end{tabular}

Abbreviations: BMI, body mass index; DBP, diastolic blood pressure; LDL-C, low-density lipoprotein cholesterol; SBP, systolic blood pressure.

Data are mean \pm s.d. unless stated otherwise.

a Definition of normal: blood pressure: SBP $<140 \mathrm{~mm} \mathrm{Hg}, \mathrm{DBP}<90 \mathrm{~mm} \mathrm{Hg}, \mathrm{BM}$

$<24 \mathrm{~kg} \mathrm{~cm}^{-2}$, abdominal circumference $<90 \mathrm{~cm}$ (men), $<85 \mathrm{~cm}$ (women), LDL-C

$<3.3 \mathrm{mmoll}^{-11}$ and FPG $<5.6 \mathrm{mmoll}^{-1}$. $^{7}$

bGroup $t$-test: compared with normal glucose metabolism, $P=0.000$

${ }^{c}$ Mann-Whitney test: compared with normal glucose metabolism, $P=0.000$.

\section{OGTT data}

Patients. A total of 5322 hypertensive patients agreed to undergo an OGTT. The characteristics of these patients are shown in Table 2. Their mean FPG level was $5.64 \mathrm{mmoll}^{-1}$ and the mean 2-h plasma glucose was $8.01 \mathrm{mmoll}^{-1}$. The glucose metabolism of these hypertensive patients determined using only FPG criteria or using either FPG criteria (in blue) or 2-h OGTT criteria is shown in Figure 1.

In addition, an analysis of the mean 2-h plasma glucose levels stratified by the FPG level (Table 3) shows that the mean 2-h plasma glucose was $>7.8 \mathrm{mmoll}^{-1}$ in patients with an FPG of $\geqslant 5.6 \mathrm{mmoll}^{-1}$. This result suggests that at least $50 \%$ of them had a 2-h plasma glucose load of $7.8 \mathrm{mmoll}^{-1}$ or more and that these patients met the criteria for abnormal glucose metabolism (DM or IGT) by OGTT criteria. ${ }^{5}$ Giving an OGTT to the patients with an FPG level $\geqslant 5.6 \mathrm{mmoll}^{-1}$ may provide higher detection rates of abnormal glucose metabolism than only performing an OGTT in those patients with an FPG level $\geqslant 6.1 \mathrm{mmoll}^{-1}$ (Table 3).
Table 2 Clinical characteristics of the subset of patients with hypertension who also underwent an oral glucose tolerance test (OGTT) $(n=5322)$

\begin{tabular}{|c|c|c|c|}
\hline & Large hospitaß & $\begin{array}{c}\text { Community } \\
\text { hospital }\end{array}$ & $\begin{array}{c}\mathrm{P} \text { - } \\
\text { value }\end{array}$ \\
\hline Number of pts, $n(\%)$ & $3366(63.2)$ & $1956(36.8)$ & - \\
\hline Male, $n(\%)$ & $1759(52.3)$ & $973(49.7)$ & 0.077 \\
\hline Age $\geqslant 60$ years, $n(\%)$ & $1458(43.3)$ & $876(44.8)$ & 0.330 \\
\hline $\mathrm{SBP}(\mathrm{mm} \mathrm{Hg})$, mean \pm s.d. & $143.27 \pm 18.48$ & $140.38 \pm 14.95$ & 0.000 \\
\hline DBP $(m m ~ H g)$, mean \pm s.d. & $86.75 \pm 12.55$ & $85.45 \pm 10.38$ & 0.000 \\
\hline BMI $\left(\mathrm{kg} \mathrm{m}^{-2}\right)$, mean \pm s.d. & $25.53 \pm 3.33$ & $25.58 \pm 3.30$ & 0.541 \\
\hline $\begin{array}{l}\text { Abdominal circumference }(\mathrm{cm}) \\
\text { mean } \pm \text { s.d. }\end{array}$ & $89.37 \pm 10.01$ & $88.95 \pm 9.49$ & 0.131 \\
\hline FPG $\left(\mathrm{mmol}^{-1}\right)$, mean \pm s.d. & $5.74 \pm 1.56$ & $5.48 \pm 1.10$ & 0.000 \\
\hline $\mathrm{TC}\left(\mathrm{mmol} \mathrm{I}^{-1}\right)$, mean $\pm \mathrm{s} . \mathrm{d}$. & $4.90 \pm 1.11$ & $5.00 \pm 1.03$ & 0.001 \\
\hline $\mathrm{TG}\left(\mathrm{mmol} \mathrm{I}^{-1}\right)$, mean $\pm \mathrm{s} . \mathrm{d}$. & $1.87 \pm 1.42$ & $1.85 \pm 1.22$ & 0.760 \\
\hline LDL-C $\left(\mathrm{mmoll}^{-1}\right)$, mean \pm s.d. & $3.16 \pm 0.98$ & $3.26 \pm 0.94$ & 0.002 \\
\hline $\operatorname{SCr}(\mu$ mol I-1), mean \pm s.d. & $82.36 \pm 30.14$ & $87.82 \pm 25.79$ & 0.000 \\
\hline eGFR ( $\mathrm{ml} \mathrm{min}-1$ per $\left.1.73 \mathrm{~m}^{2}\right)$ & $82.58 \pm 32.58$ & $79.57 \pm 29.33$ & 0.004 \\
\hline
\end{tabular}

Abbreviations: BMI, body mass index; DBP, diastolic blood pressure; eGFR, estimated glomerular filtration rate; FPG, fasting plasma glucose; LDL-C, low-density lipoprotein cholesterol; SBP, systolic blood pressure; $\mathrm{SCr}$, serum creatinine; ; TC, total cholesterol; TG, triglycerides.

Data are shown by hospital type.

a Large hospitals in China are tertiary hospitals with $>2000$ inpatient beds.

Table 3 Plasma glucose levels prior to and $2 \mathrm{~h}$ after a glucose load in an oral glucose tolerance test (OGTT) shown according to different fasting plasma glucose (FPG) levels $(n=5322)$

\begin{tabular}{lccc} 
Fasting plasma & $\begin{array}{c}\text { Number of pts } \\
\text { glucose }\end{array}$ & $\begin{array}{c}\text { Plasma glucose prior to } \\
\text { glucose load }\end{array}$ & $\begin{array}{c}\text { Plasma glucose } 2 \mathrm{~h} \\
\text { after glucose load }\end{array}$ \\
\hline$<5.6 \mathrm{mmoll}^{-1}$ & $2635(49.51)$ & $4.85 \pm 0.46$ & $7.20 \pm 1.82$ \\
$\geqslant 5.6 \mathrm{mmolI}^{-1 \mathrm{a}}$ & $2687(50.49)$ & $6.43 \pm 1.58$ & $8.79 \pm 3.10$ \\
$\geqslant 6.1 \mathrm{mmoll}^{-1}$ & $1358(25.5)$ & $7.05 \pm 2.04$ & $9.68 \pm 3.59$
\end{tabular}

alncludes all patients whose FPG was $\geqslant 5.6 \mathrm{mmol}^{-1}$; among these patients, about $50 \%$ $(1358 / 2687)$ had an FPG level of $\geqslant 6.1 \mathrm{mmoll}^{-1}$.

The proportion of patients with IGT was 53.4\% (2840/5322), and $13.6 \%(726 / 5322)$ met the criteria for DM. FPG testing of hypertensive patients detected $31.2 \%$ of patients with IGR or DM, whereas an OGTT detected $67 \%$ of such patients, thereby raising the detection rate by $35.8 \%$ (Figure 1). Thus, an OGTT doubled the IGR detection rate compared with a single FPG measurement.

BP control and glucose metabolism. Hypertensive patients with DM according to the OGTT criteria had worse SBP control than those with normal blood glucose levels (that is, normoglycemia) or with IGR (Table 4). The SBP in the NGM (normal glucose metabolism), IGR and DM groups was $141.77 \pm 16.84 \mathrm{~mm} \mathrm{Hg}$. BP control was defined as an SBP/DBP of $<140 / 90 \mathrm{mmHg}$. The proportion of patients in the subgroup with either IGR or DM who had SBP control was $34.5 \%$ and those who had DBP control was $30.6 \%$. The proportions of patients who received combination therapy in the NGM, IGR and DM groups were $39.0 \%, 43.9 \%$ and $45.2 \%$, respectively. Although more hypertensive patients with IGR received 
Table 4 The association between blood pressure level and glucose metabolism $(n=5322)^{a}$

\begin{tabular}{lcrr}
\hline & Normal glucose metabolism $(\mathrm{n}=1756)$ & IGR $(\mathrm{n}=2840)$ & DM $(\mathrm{n}=726)$ \\
\hline Systolic blood pressure $(\mathrm{mm} \mathrm{Hg})$, mean \pm s.d. & $141.77 \pm 16.84$ & $141.78 \pm 17.07$ & $144.96 \pm 19.10$ \\
Diastolic blood pressure $(\mathrm{mm} \mathrm{Hg})$, mean \pm s.d. & $86.58 \pm 11.42$ & $86.04 \pm 11.81$ & $86.40 \pm 12.75$ \\
\hline
\end{tabular}

Abbreviations: ANOVA, analysis of variance; DM, diabetes mellitus; IGR, impaired glucose regulation.

aOne-way ANOVA test was used.

combination therapy than did hypertensive patients with normal glucose metabolism $(P=0.005)$, the BP control rate was still lower in the IGR group than that in the NGM group.

Kidney damage according to glucose metabolism in the OGTT group. The prevalence of clinical proteinuria and microalbuminuria in hypertensive patients increased as glucose regulation worsened (occurring in $22.1 \%$ of patients with IGR $v$ s. $33.7 \%$ of patients with DM). Kidney damage in hypertensive patients with abnormal glucose metabolism was more common than in those with hypertension alone.

The mean eGFR in hypertensive patients with IGR was $80.77 \mathrm{ml} \mathrm{min}^{-1}$ per $1.73 \mathrm{~m}^{2}$ and in hypertensive patients with DM was $79.74 \mathrm{ml} \mathrm{min}^{-1}$ per $1.73 \mathrm{~m}^{2}$. The proportion of patients with an eGFR of $<60 \mathrm{ml} \mathrm{min}^{-1}$ per $1.73 \mathrm{~m}^{2}$ increased as impairment in glucose metabolism increased $(22.7 \%, 27.8 \%$ and $31.8 \%$ in the NGM, IGR and DM groups, respectively).

Prevalence of cardiovascular disease according to glucose metabolism. The prevalence of cardiovascular or cerebrovascular disease in hypertensive patients with IGR or DM was $17.6 \%$; the prevalence was $13.8 \%$ in normoglycemic patients (Mann-Whitney test, $P<0.001)$. Cardiovascular diseases included angina pectoris, myocardial infarction, percutaneous coronary intervention and heart failure. Cerebrovascular diseases included cerebral infarction, cerebral hemorrhage and transient cerebral ischemic attack.

Factors associated with abnormal glucose metabolism. The following factors were included in the multivariate analysis in the OGTT population $(n=5322)$ as independent variables: course of hypertension, BP control $(<$ or $\geqslant 140 / 90 \mathrm{~mm} \mathrm{Hg}$ ), proteinuria (positive or negative), eGFR ( $\leqslant$ or $>60 \mathrm{ml} \mathrm{min}^{-1}$ per $1.73 \mathrm{~m}^{2}$ ), abdominal circumference (abnormal or normal) and antihypertensive drug use (yes or no). Abnormal glucose metabolism (IGR or DM according to the OGTT criteria) was the dependent variable. The results showed that in hypertensive patients, abnormal abdominal circumference, decreased eGFR and proteinuria were significantly associated with abnormal glucose metabolism (DM or IGR) (Table 5).

\section{DISCUSSION}

Hypertension and DM are the chief components of metabolic syndrome. It has been reported that $\sim 60-70 \%$ of people who suffer from DM also have hypertension, ${ }^{3,7,8}$ and conversely, many hypertensive patients have abnormal glucose regulation. ${ }^{1}$ The rate of cardiovascular disease events doubles when high BP co-occurs with DM. ${ }^{3}$ Wang et al. ${ }^{9}$ reported 360 cases of hypertensive patients with fasting blood glucose $<5.6 \mathrm{mmoll}^{-1}$ who accepted an OGTT test, and they detected 164 cases of IGT $(45.6 \%)$ and 48 cases of DM $(13.3 \%)$. In 1166 cases of hypertensive patients in the Jiangxi province, $64.7 \%$ of patients were found to have abnormal glucose metabolism. ${ }^{10}$ In the current study, 10173 patients with essential hypertension but of unknown diabetic status were included. FPG testing revealed that $31.2 \%$ of these patients had abnormal glucose
Table 5 Factors associated with abnormal glucose metabolism in patients with essential hypertension $(n=5322)$. Results of a multivariate logistic regression analysis ${ }^{\mathrm{a}}$

\begin{tabular}{lrrrrrr}
\hline & $B$ & s.e. & Wals & Df & P-value & Exp (B) \\
\hline $\begin{array}{l}\text { Abnormal abdom- } \\
\text { inal circumference }\end{array}$ & 0.304 & 0.069 & 19.115 & 1 & 0.000 & 1.355 \\
Decreased eGFR & 0.152 & 0.045 & 11.656 & 1 & 0.001 & 1.165 \\
Proteinuria present & 0.265 & 0.090 & 8.721 & 1 & 0.003 & 1.303 \\
Constant value & -0.693 & 0.185 & 14.057 & 1 & 0.000 & 0.500 \\
\hline
\end{tabular}

Abbreviations: B, regression coefficient; $\mathrm{df}$, degree of freedom; eGFR, estimated glomerular filtration rate; $\operatorname{Exp}(\mathrm{B})$, odds ratio; Wals, $\chi^{2}$ value.

a Multivariate logistic regression analysis: the independent variables include course of

hypertension, blood pressure control $(<$ or $\geqslant 140 / 90 \mathrm{~mm} \mathrm{Hg}$ ), proteinuria (positive or negative), eGFR ( $\leqslant$ or $>60 \mathrm{ml} \mathrm{min}^{-1}$ per $1.73 \mathrm{~m}^{2}$ ), abdominal circumference (abnormal or normal) and antihypertensive drug use (yes or no).

metabolism (either IFG or DM), whereas oral glucose testing in a subgroup of 5322 patients found that the rate of abnormal glucose metabolism (that is, either IGT or DM) was 67\%, as indicated by impaired postprandial glycemia. Compared with FPG testing, an OGTT increased the detection rate by $35.8 \%$. Among the 5322 hypertensive patients, 2633 patients with a fasting blood glucose $<5.6 \mathrm{mmoll}^{-1}$ had an OGTT test. Of these, 879 patients $(33.3 \%)$ had abnormal glucose metabolism. That is, OGTTs detected $33.3 \%$ of patients with abnormal glucose metabolism, similar to the results from the aforementioned studies. Although some hypertensive patients with a fasting blood glucose $<5.6 \mathrm{mmoll}^{-1}$ refused the OGTT test, the proportion with abnormal glucose metabolism based on OGTT screening may be higher than the actual data reported in the study. An OGTT can indeed significantly improve the detection rate of abnormal glucose metabolism.

Our study also showed that for hypertensive patients with an FPG $\geqslant 5.6 \mathrm{mmoll}^{-1}$, the mean OGTT 2-h plasma glucose level was $>7.8 \mathrm{mmoll}^{-1}$. If an OGTT were conducted only in patients with FPG levels $\geqslant 6.1 \mathrm{mmoll}^{-1}$, the rate of abnormal glucose metabolism in all hypertensive patients would be underestimated. When the FPG levels were between 5.6 and $7.0 \mathrm{mmoll}^{-1}$ (that is, IFG), a significant number of patients had abnormal postprandial plasma glucose levels. IGT is associated with an increased risk of cardiovascular disease. ${ }^{8}$ Indeed, the 2010 edition of the Chinese hypertension guidelines includes the evaluation of IFG and IGT in the recommendations for the management of hypertension ${ }^{1}$ to remind clinicians to pay attention to the early evaluation of blood glucose levels in hypertensive patients.

In accordance with The American Diabetes Association Guidelines ${ }^{7}$ (current at the time we designed our study), our study defined the lower cutoffs of IFG as an FPG level $>5.6 \mathrm{mmoll}^{-1}$ and IGT as a 2-h plasma glucose level $\geqslant 7.8 \mathrm{mmoll}^{-1}$. On this basis, we found that $67 \%$ of the Chinese hypertensive patients had pre- and postprandial glucose metabolism impairments. Therefore, our study shows that, in China, the proportion of hypertensive patients with accompanying 
glucose metabolism disorders is high. Therefore, it is crucial to highlight the importance of blood glucose screening in hypertensive patients so that early effective preventative measures can be implemented. Use of the simplified OGTT would increase the detection rate of glucose metabolism abnormalities by $35.8 \%$ without increasing the patient's burden.

Hypertension with albuminuria was considered to be an early biomarker of hypertension and diabetic kidney damage. ${ }^{11}$ The kidney is an important target end-organ in hypertension, and the combination of poor BP control and poor blood glucose control has a negative impact on the kidney. It has previously been shown that albuminuria occurs in patients with coronary artery disease, regardless of whether the patient is accompanied by DM, and that albuminuria is associated with increased rates of cardiovascular disease, all-cause death and hospitalization due to heart failure. ${ }^{12}$ Our previous study carried out in patients with essential hypertension also showed that as BP levels increased, the prevalence of albuminuria increased and that the extent of urinary protein excretion was significantly different between those with good BP control and those with poor BP control $(27.1 \%$ vs. $30.2 \%, P<0.05) .{ }^{13}$ We also found that hypertensive patients with abnormal glucose metabolism had a higher prevalence of albuminuria and that the number of patients who had DM was 1.6-fold higher in those with total proteinuria than in those without proteinuria $(42.4 \%$ vs. $26.3 \%$ of patients), ${ }^{14}$ which further suggests that the coexistence of hypertension and hyperglycemia is likely to do more harm to the kidneys. ${ }^{14}$ Our current study results support the observation that worsening of glucose regulation in hypertensive patients is associated with declining kidney function: the eGFR in patients with hypertension and no glucose metabolism disorders was $82.9 \mathrm{ml} \mathrm{min}^{-1}$ per $1.73 \mathrm{~m}^{2}$ vs. those with hypertension and IGR, which was $80.77 \mathrm{ml} \mathrm{min}^{-1}$ per $1.73 \mathrm{~m}^{2}$, and those with hypertension and DM, which was $79.74 \mathrm{ml} \mathrm{min}^{-1}$ per $1.73 \mathrm{~m}^{2}$ (although differences between the groups were not significant). Moreover, we found that hypertensive patients with abnormal glucose metabolism had higher rates of albuminuria than did those with normoglycemia $33.7 \%$ of patients with DM vs. $22.1 \%$ of patients with IGR vs. $15.8 \%$ of normoglycemic patients). Overall, $27.9 \%$ of patients who underwent an OGTT in our study had albuminuria, similarly to our previously reported results $(28.8 \%){ }^{13}$

Finally, the multivariate logistic regression analysis indicated that a large abdominal circumference, proteinuria and impaired kidney function (assessed by eGFR) were independently associated with abnormal glucose metabolism. This finding suggests that obese hypertensive patients should be monitored for changes in their glucose metabolism and that an OGTT should be administered. In addition, clinicians treating hypertensive patients should be aware of the risk of kidney organ damage in the overall evaluation of patients with hypertension so that proper treatment may be provided.

The limitations of this study are as follows:

- Not all patients with hypertension included in our study underwent the OGTT, which suggests that the OGTT results may have overestimated the prevalence of IGT in these patients.

- Participating sites were selected by geographical location and not randomly selected.

\section{CONCLUSION}

A total of $67 \%$ of hypertensive patients in China have DM or are at an increased risk of DM (IFG/IGT), which has a close association with microalbuminuria. They are therefore more likely to suffer from kidney damage, as well as cardiovascular or cerebrovascular diseases. The use of an OGTT can raise the detection of glycemic disorders by $35.8 \%$, and the detection rate is maximized if an OGTT is implemented in those with FPG levels $\geqslant 5.6 \mathrm{mmoll}^{-1}$. We conclude that testing FPG levels and using the simplified OGTT can help clinicians to evaluate the risk of DM in patients with hypertension and to take early and effective preventative measures to improve patient outcomes.

\section{CONFLICT OF INTEREST}

The authors declare no conflict of interest.

\section{ACKNOWLEDGEMENTS}

We thank Tracy Harrison of inScience Communications, Springer Healthcare, who provided assistance with the English language and technical editing. This assistance was funded by the Bayer Healthcare (China). We also thank all of the hospitals and experts who participated in the study. The following list is arranged according to alphabetical order of the hospital's Chinese name: Peking University Third Hospital, Wei Gao, Zhaoping Li; Beijing Jishuitan Hospital, Xingshan Zhao, Zhan Ma; Beijing Sixth Hospital, Xiaoping Xiang, Hanjun Yuan; Beijing Hospital, Qin He, Hua Wang; Daping Hospital of Chongqing, Zhiming Zhu, Yinxing Ni; Daqing Oilfield General Hospital of Heilongjiang Province, Jinping Wang; Southwest Hospital of The Third Military Medical University, Liangyi Si, Qiang Xu; Tangdu Hospital of The Fourth Military Medical University, Lianyou Zhao, Yongfei Ai; Huashan Hospital of Fudan University, Yong Li, Weilin Qi; Hebei Provincial People's Hospital, Shuren Li; The Second Hospital of Hebei Medical University, Yuming Hao; Henan Provincial People's Hospital, Hao Wang, Shuxian Cui; Huadong Hospital, Yu Zhang; Tongji Hospital of Huazhong University of Science and Technology, Xiaomei Guo, Hua Liao; Shanghai Eighth Hospital of Jiangsu University, Qingping Chang, Changwu Ruan; Civil Aviation General Hospital, Wen Yang; Inner Mongolia People's Hospital, Yajun Han; The First Affiliated Hospital of Nanchang University, Yunfeng Wei, Xiaoping Peng; Putuo Distric Center Hospital, Huigen Jin; The Affiliated Hospital of Medical College Qingdao University, Shanglang Cai, Wenzhong Zhang; The First Hospital of Shanxi Medical University, Shuiwang Zhang; The Sixth Hospital of Shanghai, Qing Zhao; The First Hospital of Shanghai, Ying Wu; Beijing Chaoyang Hospital Affiliated to Capital Medical University, Mingming Gao, Yuan Zhang; Beijing Tiantan Hospital Affiliated to Capital Medical University, Fenghe Du, Hongtao Gong; Fuxin Hospital Affiliated to Capital Medical University, Ming Yang, Zhaoxia Yin; Xuanwu Hospital Affiliated to Capital Medical University, Qi Hua, Yinzhen Chen; West China Hospital of Sichuan University, Xiaoping Chen, Qing Peng; Tianjin Medical University General Hospital, Yuemin Sun; Xiluoyuan Community Health Center, Ling Tian, Hongwei Xu; The First Hospital of Xiamen, Qiang Xie; Yueyang Hospital, Deyu Fu; Second Affiliated Hospital Zhejiang University College of Medicine, Meixiang Xiang, Zhenni Jiang; Second Affiliated Hospital Zhejiang University College of Medicine, Geng Xu, Ying Zhou; The First Affiliated Hospital of Zhengzhou University, Zhenwen Huang, Xin Fu; and Xiangya Hospital of Central-South University, Tianlun Yang, Qilin Ma.

Author contributions: Ningling Sun contributed to discussions and reviewed and edited the manuscript. Hongyi Wang performed the statistical analyses and wrote parts of the manuscript. Yuemin Sun, Xiaoping Chen, Hao Wang, Lianyou Zhao, Jinping Wang and Zhiming Zhu contributed to the data collection. All the authors read and approved the final manuscript.

1 Liu LS. Writing Group of 2010 Chinese Guidelines for the Management of Hypertension. 2010 Chinese guidelines for the management of hypertension. Zhonghua Xin Xue Guan Bing Za Zhi 2011: 39: 579-615.

2 He J, Gu D, Chen J, Wu X, Kelly TN, Huang JF, Chen JC, Chen CS, Bazzano LA Reynolds K, Whelton PK, Klag MJ. Premature deaths attributable to blood pressure in China: a prospective cohort study. Lancet 2009; 374: 1765-1772.

3 American Diabetes Association. Hypertension management in adults with diabetes Diabetes Care 2004; 27 (Suppl 1), S65-S67. 
4 American diabetes association. Diagnosis and classification of diabetes mellitus. Diabetes Care 2012; 35 (Suppl 1), S64-S71.

5 Hu DY, Pan CY, Yu JM. China Heart Survey Group. The relationship between coronary artery disease and abnormal glucose regulation in China: the China Heart Survey. Eur Heart J 2006; 27: 2573-2579.

6 Ma YC, Zuo L, Chen JH, Luo Q, Yu XQ, Li Y, Xu JS, Huang SM, Wang LN, Huang W, Wang $\mathrm{M}, \mathrm{Xu} \mathrm{GB}$, Wang HY. Modified glomerular filtration rate estimating equation for Chinese patients with chronic kidney disease. J Am Soc Nephrol 2006; 17: 2937-2944.

7 American Diabetes Association. Standards of medical care in diabetes. Diabetes Care 2005; 28 (Suppl 1), S4-S36.

8 Geiss LS, Rolka DB, Engelgau MM. Elevated blood pressure among U.S. adults with diabetes, 1988-1994. Am J Prev Med 2002; 22: 42-48.

9 Wang QR, Wang RY, Wang R. Glucose metabolism state in patients with normal fasting blood glucose and essential hypertension. Chin J Integrative Med Cardio/cerebrovascular Dis 2010; 8: 268-269.
10 Zou F, Lai XY. Study of glucometabolic abnormality and risk factors in patients with essential hypertension(EH)in Jiangxi Province. Chin J Diabetes 2009; 17: $116-118$.

11 de Zeeuw D. Albuminuria, just a marker for cardiovascular disease, or is it more? J Am Soc Nephrol 2005; 16: 1883-1885

12 Gerstein HC, Mann JF, Yi Q, Zinman B, Dinneen SF, Hoogwerf B, Hallé JP, Young J, Rashkow A, Joyce C, Nawaz S, Yusuf SHOPE Study Investigators. Albuminuria and risk of cardiovascular events, death, and heart failure in diabetic and nondiabetic individuals. JAMA 2001; 286: 421-426.

13 Sun NL, Wang HY, Zhu DL, Liao YH, Lin SG, Chen XP. Association between albuminuria and blood pressure level in patients with essential hypertension. Chin $J$ of Nephro 2010; 26: 762-765.

14 Sun NL, Wang HY, Liao YH, Zhu DL, Zhang TJ, Chen XP, Pan CY, Zhu JR. Relationship between albuminuria and dysglycaemia in patients with essential hypertension. Chin $\mathrm{J}$ of Hypertens 2010; 18: 1138-1141. 